\title{
Empirical Likelihood for Right Censored Lifetime Data
}

\author{
Shuyuan $\mathrm{He}^{1 *}$, Wei Liang ${ }^{2 *}$, Junshan Shen ${ }^{3 *}$ and Grace Yang ${ }^{4}$ * \\ Capital Normal University ${ }^{1}$, Xiamen University ${ }^{2}$, \\ Peking University ${ }^{3}$, University of Maryland ${ }^{4}$
}

November 2, 2018

\begin{abstract}
This paper considers the empirical likelihood (EL) construction of confidence intervals for a linear functional $\theta$ based on right censored lifetime data. Many of the results in literature show that $-2 \log \left(\right.$ empirical likelihood ratio) has a limiting scaled- $\chi_{1}^{2}$ distribution, where the scale parameter is a function of the unknown asymptotic variance. The scale parameter has to be estimated for the construction. Additional estimation would reduce the coverage accuracy for $\theta$. This diminishes a main advantage of the EL method for censored data. By utilizing certain influence functions in an estimating equation, it is shown that under very general conditions, $-2 \log$ (EL ratio) converges weakly to a standard $\chi_{1}^{2}$ distribution and thereby eliminates the need for estimating the scale parameter. Moreover, a special way of employing influence functions eases the otherwise very demanding computations of the EL method. Our approach yields smaller asymptotic variance of the influence function than those comparable ones considered by Wang \& Jing (2001) and Qin \& Zhao (2007). Thus it is not surprising

\footnotetext{
${ }^{*}$ Research of authors 1, 2 and 3 were Supported by National Natural Science Foundation of China(11171230, 10801003). Research of author 4 was supported by the US National Science Foundation, while working at the Foundation.
} 
that confidence intervals using influence functions give a better coverage accuracy as demonstrated by simulations.

Key words and phrases. Empirical likelihood, Right censored lifetimes, Influence function, Parameter estimation, Confidence intervals

AMS 1991 subject classifications. Primary 62H99; Secondary 62H05.

\section{Introduction}

Let $Y$ be a non-negative random variable with distribution function $F(y)=P[Y \leq y]$.

Let $C$ be a nonnegative random variable with distribution function $G(x)=P[C \leq x]$ and $C$ is independent of $Y$. Instead of $Y$, we observe $Z=\min (Y, C)$ and the indicator $\delta=I[Y \leq C]$ of the event $[Y \leq C]$. Both $F$ and $G$ are assumed continuous but unknown. In this paper we use the empirical likelihood method with right-censored data to study the problem of constructing confidence intervals for $\theta$, a functional of the distribution $F$ defined by $\mathrm{E} g(Y, \theta)=0$. For example, if $g(x, \theta)=\xi(x)-\theta$, for some function $\xi$ we get

$$
\theta=\mathrm{E} \xi(Y)=\int_{0}^{\infty} \xi(x) \mathrm{d} F(x) .
$$

The problem of estimating $\theta$ in (1.1) with a sample of $n$ i.i.d. observations of $(Z, \delta)$ has been studied by many authors. If $\xi(Y)=I[Y \leq y]$ for a fixed $y$, then $\theta=F(y)$. A well-known nonparametric maximum likelihood and asymptotically optimal estimator of $F(y)$ is the Kaplan-Meier (KM) estimator $F_{n}$ as defined in (2.3). A natural estimator of $\theta$ is

$$
\theta_{n}=\int_{0}^{\infty} \xi(s) \mathrm{d} F_{n}(s) .
$$

For an arbitrary function $\xi$, several authors, e.g. Yang (1994) have shown that under the condition of finite second moment,

$$
\int_{0}^{\infty} \frac{\xi^{2}(s)}{\bar{G}(s)} \mathrm{d} F(s)<\infty,
$$


the asymptotic distribution of $\sqrt{n}\left(\theta_{n}-\theta\right)$, as $n$ goes to infinity, is normal $N\left(0, \sigma^{2}\right)$, where

$$
\sigma^{2}=\int_{0}^{\infty} \frac{(\bar{F}(s) \xi(s)-\psi(s))^{2}}{\bar{F}^{2}(s) \bar{G}(s)} \mathrm{d} F(s), \psi(s)=\int_{s}^{\infty} \xi(x) \mathrm{d} F(x), s \geq 0,
$$

and $\bar{G}=1-G, \bar{F}=1-F$.

Confidence intervals for $\theta$ can be constructed using the asymptotic normal distribution $N\left(0, \sigma^{2}\right)$. Alternatively, the EL method can be used as to be investigated in this paper. Employing either method, one needs to deal with a rather complicated form of the asymptotic variance $\sigma^{2}$. Among other things, it is computationally demanding.

To use the normal distribution $N\left(0, \sigma^{2}\right)$, it is necessary to estimate the unknown variance $\sigma^{2}$. Stute (1996) proposed a jackknife estimator to replace $\sigma^{2}$ in the calculation. Although any consistent estimator $\sigma_{n}^{2}$ of $\sigma^{2}$ can be used, the convergence rate of $\sigma_{n}^{2}$ is generally unknown. Substitution by the estimate $\sigma_{n}^{2}$ tends to reduce the coverage accuracy for $\theta$ as compare to the case of known $\sigma^{2}$.

The usefulness of the EL method for constructing confidence interval/regions has been well established in a wide variety of situations, see e.g., DiCiccio et al. (1991) and Chen (1994), and an extensive literature review in Owen (2001) to that day. Let $R(\theta)$ denote the EL ratio function of a one-dimensional parameter $\theta$ for $n$ i.i.d "complete" observations. Owen (1988) proved that under certain regularity conditions, $-2 \log R(\theta)$ converges to a chi-squared distribution with one degree of freedom. The EL method gives confidence intervals for $\theta$ as $\left\{\theta:-2 \log R(\theta) \leq c_{1-\alpha}\right\}$, where $c_{1-\alpha}$ is the $(1-\alpha)$ th quantile of the $\chi_{1}^{2}$ distribution. Here the construction of confidence intervals does not require estimation of asymptotic variance. In view of a complicated variance formula in (1.4), this would have provided a welcome method for censored data. However, as far as we know, for censored data, the asymptotic standard chi-squared distribution holds only in some special cases see, e.g. Owen (Chapter 6, 2001). More recent literature shows that most of the asymptotic distributions involve weights which are functions of unknown variances or covariance matrices. This is the case, for example, in the 
following papers. Li and Wang (2003) studied right-censored regression models, Ren (2008) used weighted EL under a variety of censoring models, Wang \& Jing (2001) and Qin \& Zhao (2007) estimated functionals $\theta$, and Hjort\& McKeague \& van Keilegom, in their extension of the scope of the EL method (2009), obtained an asymptotic distribution (Theorem 2.1) which is a sum of weighted chi-squared distributions with unknown weights. Therefore, using these results to construct confidence intervals for $\theta$ still require an additional estimation of the unknown $\sigma^{2}$. This diminishes a main advantage of the EL method for censored data.

The EL ratio $R(\theta)$ is obtained by utilizing auxiliary information on $\theta$ through a set of estimating equations. In this paper, we show that by using certain influence functions with a special construction of estimating equations in the EL ratio, the asymptotic distribution of $-2 \log R(\theta)$ of the functional $\theta$ in (1.1) is a standard $\chi_{1}^{2}$ without involving any unknown scale parameter. Our approach transfers the problem of estimating $\sigma^{2}$ to the influence functions. As a result, it also significantly simplifies the often intensive computations of the EL method for censored data.

Our work is motivated by the work of Wang \& Jing (2001) and Qin \& Zhao (2007). Wang \& Jing (2001) obtained an EL ratio by first finding an estimating equation for a certain complete sample and then modifying the estimating equation for the right censored sample. The resulting estimating equation is a sum instead of a product (inherent of the product limit estimator). With this approach, Wang \& Jing (2001) use the estimating function $M_{1}(Z, \delta, \theta)$ for $\theta$ (see (3.7) ) and Qin \& Zhao (2007) use $M_{2}(Z, \delta, \theta)$ (see (3.8)) for estimating the mean residual life $\mathrm{E}\left(Y-t_{0} \mid Y \geq t_{0}\right)$ at age $t_{0}$. However, both of these papers obtain an asymptotic scaled $\chi_{1}^{2}$ distribution for $-2 \log R(\theta)$.

Instead of $M_{1}$ and $M_{2}$, we use influence functions. We compute the influence functions $W(Z, \delta, \theta)$ of

$$
\mu_{n}=\int_{0}^{\infty} g(x, \theta) \mathrm{d} F_{n}(x)
$$


as defined by (3.6), where $F_{n}$ is the Kaplan-Meier estimator. The influence functions $W^{\prime} s$ are to be utilized to construct an estimation function for the EL method. Numerous examples of the function $g$ are given in Section 2 .

The paper is organized as follows. Preliminaries assumptions and examples of $\theta$ and $g$ are given in Section 2. The influence function $W(Z, \delta, \theta)$ are given in Section 3. It is shown in Theorem 3.1 that asymptotically $\sqrt{n}\left(\mu_{n}-\mu\right)$ is a partial sum of $n$ independent influence functions $W\left(Z_{j}, \delta_{j}, \theta\right)$ (an IID representation), or is asymptotically linear. Here $\mu$ denotes $\operatorname{Eg}(Y, \theta)$ and the the condition $\operatorname{Eg}(X, \theta)=0$ is not imposed in Section 3. These results are general for any $\xi(x)$ having finite second moment (1.3) and no restrictions are placed on the upper boundaries of $X$ and $C$.

An IID representation of the Kaplan-Meier estimator has been obtained by many authors e.g., Lo \& Singh (1986), Stute \& Wang (1993) and Chen \& Lo (1997) using different approaches, under different conditions and in different forms. See Yang (1997) and references therein. Here, we use an influence function $W(Z, \delta, \theta)$ of $\theta_{n}$ in $(1.2)$ obtained in He \& Huang(2003). We show that the variance of the influence function is smaller than that of $M_{1}$ and $M_{2}$ (see Remark 3.1 in Section 3), which results in an improvement of the asymptotic coverage accuracies of $\theta$. In Section 4 , estimation of the influence functions is carried out in Theorem 4.1. The weak convergence of $-2 \log R(\theta)$ to the standard $\chi_{1}^{2}$ distribution without any scale parameter is proved in Theorem 4.2 which justifies the EL construction of confidence intervals for censored data. In Section 5, simulation comparison of the new method with that of the scaled $\chi_{1}^{2}$ distribution is presented. The amount of improvement depends on the form of $\theta$. For survival function or mean, the coverage ratios computing from the traditional normal approximation and the EL method are about the same. The EL method performs better for more complicated $\theta$. Most of the proofs are relegated to the Appendix. 


\section{Preliminaries, Assumptions and Examples}

For any right continuous monotone function $h(x)$, let $h(x-)$ or $h_{-}(x)$ denote the left continuous version of $h(x)$ and the curly brackets $h\{x\}$ denote the difference $h(x)-$ $h(x-)$. Then $h\{x\}=\mathrm{d} h(x)$. For any cumulative distribution function $F$, let $\bar{F}=1-F$. Assume that

$$
F(x)=P(Y \leq x), \quad G(x)=P(C \leq x) \text { and } H(x)=P(Z \leq x)
$$

are continuous cumulative distributions of $Y, C$ and $Z=\min (Y, C)$, respectively. Let $\left[0, b_{H}\right]$ be the range of $H$, where

$$
b_{H}=\sup \{x: H(x)<1\} \text {. }
$$

$b_{F}$ and $b_{G}$ are similarly defined for $F$ and $G$. Then $b_{H}=\min \left(b_{F}, b_{G}\right)$.

Given a sample of $n$ i.i.d. random vectors $\left(Z_{i}, \delta_{i}\right), i=1,2, \cdots, n$, of $(Z, \delta)$, their empirical distribution functions are given by:

$$
\begin{aligned}
& H_{n}^{1}(x)=\frac{1}{n} \sum_{j=1}^{n} I\left[Z_{j} \leq x, \delta_{j}=1\right], \\
& H_{n}^{0}(x)=\frac{1}{n} \sum_{j=1}^{n} I\left[Z_{j} \leq x, \delta_{j}=0\right], \\
& H_{n}(x)=H_{n}^{0}(x)+H_{n}^{1}(x)=\frac{1}{n} \sum_{j=1}^{n} I\left[Z_{j} \leq x\right] .
\end{aligned}
$$

Asymptotic optimal nonparametric estimators of $F(x)$ and $G(x)$ are the well-known Kaplan-Meier estimators given by

$$
F_{n}(x)=1-\prod_{s \leq x}\left[1-\frac{H_{n}^{1}\{s\}}{\bar{H}_{n}(s-)}\right] \text { and } \quad G_{n}(x)=1-\prod_{s \leq x}\left[1-\frac{H_{n}^{0}\{s\}}{\bar{H}_{n}(s-)}\right]
$$

respectively, where an empty product is set equal to one. It can be checked that for all $x$

$$
\bar{H}_{n}(x)=\bar{F}_{n}(x) \bar{G}_{n}(x) .
$$


Applying (2.3) and (2.4) we get

$$
\mathrm{d} F_{n}(x)=F_{n}(x)-F_{n}(x-)=\bar{F}_{n}(x-) \frac{\mathrm{d} H_{n}^{1}(x)}{\bar{F}_{n}(x-) \bar{G}_{n}(x-)} .
$$

It follows that

$$
\mathrm{d} H_{n}^{1}(x)=\bar{G}_{n}(x-) \mathrm{d} F_{n}(x), \quad \mathrm{d} H_{n}^{0}(x)=\bar{F}_{n}(x-) \mathrm{d} G_{n}(x) .
$$

Put

$$
\begin{aligned}
& H^{0}(x)=P(Z \leq x, \delta=0), \quad H^{1}(x)=P(Z \leq x, \delta=1), \text { and } \\
& \bar{H}(x)=P(Z>x) .
\end{aligned}
$$

Then

$$
\begin{aligned}
& H^{0}(x)=\mathrm{E} H_{n}^{0}(x)=\int_{0}^{x} \bar{F}(s) \mathrm{d} G(s), \\
& H^{1}(x)=\mathrm{E} H_{n}^{1}(x)=\int_{0}^{x} \bar{G}(s) \mathrm{d} F(s), \\
& \bar{H}(x)=\mathrm{E} \bar{H}_{n}(x)=\bar{F}(x) \bar{G}(x) .
\end{aligned}
$$

Here and after, the integral sign $\int_{a}^{b}$ stands for $\int_{(a, b]}$ and $\int$ stands for $\int_{0}^{\infty}$.

Examples of $\theta$ and $g(x, \theta)$

1. $g(x, \theta)=I[x>y]-\theta$ with $y$ fixed. Then

$$
\mathrm{E} g(Y, \theta)=\int(I[x>y]-\theta) \mathrm{d} F(x)=0 .
$$

Solving this equation yields $\theta=\bar{F}(y)$, the survival function of $Y$.

2. $g(x, \theta)=x^{k}-\theta$. Then $\theta=\mathrm{E} Y^{k}$, the $k$ th moments of $Y$.

3. $g(x, \theta)=\left(x-t_{0}-\theta\right) I\left[x \geq t_{0}\right]$ with $t_{0}$ fixed. Then

$$
\theta=\mathrm{E}\left(Y-t_{0} \mid Y \geq t_{0}\right)=\frac{\mathrm{E}\left(Y-t_{0}\right) I\left[Y \geq t_{0}\right]}{P\left(Y \geq t_{0}\right)},
$$

the mean residual life of $Y$. 
4. $g(x, \theta)=x(I[x>y]-\theta)$. Then

$$
\theta=\frac{1}{\operatorname{E} Y} \int_{y}^{\infty} s \mathrm{~d} F(s),
$$

the length biased survival function of $Y$. See Vardi (1982), for example.

5. $g(x, \theta)=x^{2}-\theta x$. Then

$$
\theta=\frac{1}{\operatorname{EY}} \int_{0}^{\infty} x^{2} \mathrm{~d} F(x)
$$

the mean of the length-biased lifetime.

6. $g(x, \theta)=x^{2}-2 \theta x$. Then

$$
\theta=\frac{1}{2 \mathrm{E} Y} \int_{0}^{\infty} x^{2} \mathrm{~d} F(x)
$$

the mean of the length biased residual lifetime.

7. $g(x, \theta)=I[x \leq \theta]-p$ with $p \in(0,1)$. Then $\theta=F^{-1}(p)$, the $p$ th quantile of $Y$.

Examples (4)-(6) often appear in renewal processes and their applications.

\section{Influence function of $\mu_{n}$}

Throughout Section 3, $\theta$ is a fixed value. Then it is convenient to suppress $\theta$ in the exposition, by setting $\xi(x)=g(x, \theta), \mu=\operatorname{E} g(X, \theta)$, and

$$
\mu_{n}=\int \xi(x) \mathrm{d} F_{n}(x)=\int \frac{\xi(x)}{\bar{G}_{n}(x-)} \mathrm{d} H_{n}^{1}(x) .
$$

Likewise, set $W=W(Z, \delta)=W(Z, \delta, \theta)$.

In Theorem 3.1, we prove that the estimator $\mu_{n}$ for $\mu$ is asymptotic linear. That is, there is a function $W=W(Z, \delta)$, such that $\mathrm{E} W=0, \operatorname{Var}(W)<\infty$ and

$$
\sqrt{n}\left(\mu_{n}-\mu\right)=\frac{1}{\sqrt{n}} \sum_{i=1}^{n} W\left(Z_{i}, \delta_{i}\right)+o_{p}(1) .
$$

The function $W(Z, \delta)$ is defined with respect to the true distributions $(F, G)$. Following literature, we call $W\left(Z_{i}, \delta_{i}\right)$ the $i$-th influence function of $\mu_{n}$. See, for e.g. van der Vaart (1998) or Tsiatis (2006). 
Theorem 3.1 will be proved by first establishing a similar result for the truncated $W\left(Z_{i}, \delta_{i}\right)$ as defined in (3.3). Let $\xi_{b}(x)=\xi(x) I[x \leq b]$ be the restriction of $\xi(x)$ on $(-\infty, b]$, where $b<b_{H}$ is an arbitrarily chosen constant. By similar truncation, put

$$
\mu_{b}=\int \xi_{b}(x) \mathrm{d} F(x), \quad \psi_{b}(s)=\int_{x \geq s} \xi_{b}(x) \mathrm{d} F(x), \quad \bar{\delta}_{i}=I\left[Y_{i}>C_{i}\right]=1-\delta_{i} .
$$

We consider the i.i.d. random variables,

$$
W_{i}(b)=\frac{\xi_{b}\left(Z_{i}\right) \delta_{i}}{\bar{G}\left(Z_{i}\right)}-\mu_{b}+\frac{\bar{\delta}_{i}}{\bar{H}\left(Z_{i}\right)} \psi_{b}\left(Z_{i}\right)-\int \psi_{b}(s) \frac{I\left[Z_{i} \geq s\right]}{\bar{H}^{2}(s)} \mathrm{d} H^{0}(s),
$$

for $i=1, \cdots, n$.

Under finite variance condition (1.3), it can be calculated that,

$$
\begin{aligned}
\mathrm{E} \frac{\xi_{b}\left(Z_{i}\right) \delta_{i}}{\bar{G}\left(Z_{i}\right)} & =\mu_{b}, \quad \mathrm{E} W_{i}(b)=0, \\
\operatorname{Var}\left(W_{i}(b)\right) & =\int \frac{\xi_{b}^{2}(s)}{\bar{G}(s)} \mathrm{d} F(s)-\mu_{b}^{2}-\int \frac{\psi_{b}^{2}(s)}{\bar{F}(s) \bar{G}^{2}(s)} \mathrm{d} G(s) .
\end{aligned}
$$

As $b$ approaches the upper bound $b_{H}$, we have

$$
\begin{gathered}
\mu_{b} \rightarrow \mu, \quad \psi(s)=\lim _{b \rightarrow b_{H}} \psi_{b}(s)=\int_{x \geq s} \xi(x) \mathrm{d} F(x), \quad \text { and } \\
W_{i}=\lim _{b \rightarrow b_{H}} W_{i}(b)=\frac{\xi\left(Z_{i}\right) \delta_{i}}{\bar{G}\left(Z_{i}\right)}-\mu+\frac{\bar{\delta}_{i}}{\bar{H}\left(Z_{i}\right)} \psi\left(Z_{i}\right)-\int \psi(s) \frac{I\left[Z_{i} \geq s\right]}{\bar{H}^{2}(s)} \mathrm{d} H^{0}(s),
\end{gathered}
$$

for $i=1, \cdots, n$. The $W_{i}^{\prime} s$, for $i=1, \cdots, n$, are i.i.d. random variables.

Wang \& Jing (2001) use the estimating function based on

$$
M_{1}(Z, \delta, \theta)=\frac{\xi(Z) \delta}{\bar{G}(Z)}-\theta
$$

to estimate $\theta$ in (1.1). Qin \& Zhao (2007) used the estimating function based on

$$
M_{2}(Z, \delta, \theta)=\frac{g(Z, \theta) \delta}{\bar{G}(Z)}
$$

where $g(x, \theta)=\left(x-t_{0}-\theta\right) I\left[x \geq t_{0}\right]$, to estimate the mean residual life $\theta=\mathrm{E}\left(Y-t_{0} \mid Y \geq\right.$ $\left.t_{0}\right)$ at a specified age $t_{0}$. This case is covered in our formulation, see example (3) in 
Section 2. Comparing with $M_{1}$ and $M_{2}$, our $W_{i}^{\prime} s$ contain two additional terms. Note that $W_{i}^{\prime} s$ are not observable random variables and whose estimation will be addressed in Section 4.

Under finite variance condition (1.3), applying the dominated convergence theorem and the Lebesgue-Stieltjes integration by parts, we obtain

$$
\begin{aligned}
\mathrm{E} \frac{\xi\left(Z_{i}\right) \delta_{i}}{\bar{G}\left(Z_{i}\right)} & =\mu, \quad \mathrm{E} W_{i}=0 \\
\operatorname{Var}\left(W_{i}\right) & =\int \frac{\xi^{2}(s)}{\bar{G}(s)} \mathrm{d} F(s)-\mu^{2}-\int \frac{\psi^{2}(s)}{\bar{F}(s) \bar{G}^{2}(s)} \mathrm{d} G(s) \\
& =\int \frac{(\bar{F}(s) \xi(s)-\psi(s))^{2}}{\bar{F}^{2}(s) \bar{G}(s)} \mathrm{d} F(s) .
\end{aligned}
$$

Remark 3.1 Formulas (3.6) are (3.10) are obtained in He \& Huang (2003), and (3.11) is given in Yang (1994). Under condition (1.3), it can be shown that (3.10) and (3.11) are equal. The variance of $W_{i}$ is smaller than that of $M_{1}$ and $M_{2}$ defined in (3.7) and (3.8). The variance of the latter two equals

$$
\int \frac{\xi^{2}(s)}{\bar{G}(s)} d F(s)-\mu^{2}
$$

with the corresponding choices of $\xi(z)=g(z, \theta)$, in $M_{1}$ and $M_{2}$.

We proceed to prove Theorem 3.1. For the restricted $W_{i}(b)^{\prime} s$, the following lemma is taken from (3.11) of He \& Huang (2003).

Lemma 3.1 Let $F$ and $G$ be continuous. For each $\theta$ fixed, set $\xi(x)=g(x, \theta)$. Assume that $\int \xi^{2}(x) d F(x)<\infty$ and $b<b_{H}$. Let $\xi_{b}(x)$ be the restriction of $\xi$ on $(0, b]$ for $b<b_{H}$. Then, as $n \rightarrow \infty$,

$$
\sqrt{n} \int \xi_{b}(x) d\left(F_{n}(x)-F(x)\right)=\frac{1}{\sqrt{n}} \sum_{i=1}^{n} W_{i}(b)+o_{p}(1) .
$$

The following result will be used repeatedly and for easy reference, it is stated in Lemma 3.2. Its proof is given the appendix. 
Lemma 3.2 For $b<b_{H}$, let $\left\{h_{n}(b)\right\}$ be a random sequence such that $h_{n}(b) \rightarrow h(b)$ in distribution as $n \rightarrow \infty$, and $h(b)=o_{p}(1)$ as $b \rightarrow b_{H}$. As $n \rightarrow \infty$, if $V_{n}=O_{p}(1)$ and the random sequence $\left\{S_{n}\right\}$ can be written as $S_{n}=o_{p}(1)+V_{n} h_{n}(b)$ for any $b<b_{H}$, then $S_{n}=o_{p}(1)$.

Remark 3.2 In what follows, $h_{n}(b)$ is used as a generic notation to denote any random sequence $\left\{h_{n}(b)\right\}$ that satisfies the assumptions of Lemma 3.2. This simplifies many of the statements later. For example, under condition (1.3) and $b<b_{H}$, put

$$
h_{n}(b)=\int_{b}^{b_{H}} \frac{\xi^{2}(s)}{\bar{G}^{2}(s)} d H_{n}^{1}(s) .
$$

Then, by the SLLN and (2.8)

$$
\begin{aligned}
\lim _{n \rightarrow \infty} h_{n}(b) & =\lim _{n \rightarrow \infty} \int_{b}^{b_{H}} \frac{\xi^{2}(s)}{\bar{G}^{2}(s)} d H_{n}^{1}(s)=\int_{b}^{b_{H}} \frac{\xi^{2}(s)}{\bar{G}^{2}(s)} d H^{1}(s) \\
& =\int_{b}^{b_{H}} \frac{\xi^{2}(s)}{\bar{G}^{2}(s)} \bar{G}(s) d F(s)=h(b) \rightarrow 0, \text { as } b \rightarrow b_{H} .
\end{aligned}
$$

Theorem 3.1. Let $W_{i}$ be given by (3.6). Suppose $F$ and $G$ are continuous, and for each fixed $\theta$ set $\xi(x)=g(x, \theta)$. Then under condition (1.3) as $n \rightarrow \infty$,

$$
\sqrt{n} \int \xi(x) d\left(F_{n}(x)-F(x)\right)=\frac{1}{\sqrt{n}} \sum_{i=1}^{n} W_{i}+o_{p}(1) .
$$

Proof. For $b<b_{H}$, put $\bar{\xi}_{b}=\xi(x)-\xi_{b}(x)=\xi(x) I[x>b]$. Decompose the following difference as

$$
\sqrt{n} \int \xi(x) \mathrm{d}\left(F_{n}(x)-F(x)\right)-\frac{1}{\sqrt{n}} \sum_{i=1}^{n} W_{i} \equiv J_{1}(b)+J_{2}(b)-J_{3}(b),
$$

where

$$
\begin{aligned}
& J_{1}(b)=\sqrt{n} \int \xi_{b}(x) \mathrm{d}\left(F_{n}(x)-F(x)\right)-\frac{1}{\sqrt{n}} \sum_{i=1}^{n} W_{i}(b) \\
& J_{2}(b)=\sqrt{n} \int \bar{\xi}_{b}(x) \mathrm{d}\left(F_{n}(x)-F(x)\right) \\
& J_{3}(b)=\frac{1}{\sqrt{n}} \sum_{i=1}^{n}\left(W_{i}-W_{i}(b)\right)
\end{aligned}
$$


Let $\bar{\psi}_{b}(x)=\int_{s \geq x} \bar{\xi}_{b}(s) \mathrm{d} F(s)$. It follows that

$$
\bar{\psi}_{b}^{2}(x) \leq \bar{F}(x)^{2} \int_{s \geq x} \bar{\xi}_{b}^{2}(s) \mathrm{d} F(s)
$$

By Lemma 3.1, $J_{1}(b)=o_{p}(1)$. We shall show that $J_{2}(b)=h_{n}(b)$ and $J_{3}(b)=h_{n}(b)$ as $n \rightarrow \infty$. Applying Corollary 1 of Yang (1994), $J_{2}(b)$ converges weakly to $N\left(0, \bar{\sigma}_{b}^{2}\right)$, where $\bar{\sigma}_{b}^{2}$ is similarly defined as in (3.12) with $\xi$ and $\psi$ replacing by their restrictions $\bar{\xi}_{b}$ and $\bar{\psi}_{b}$ respectively.

Now

$$
\bar{\sigma}_{b}^{2} \leq \int \frac{\bar{\xi}_{b}^{2}(x)}{\bar{G}(x)} \mathrm{d} F(x) \rightarrow 0, \text { as } b \rightarrow b_{H} .
$$

Therefore $J_{2}(b)$ converges to $h(b)=Z_{0} \bar{\sigma}_{b}$ in distribution, and $h(b)=o_{p}(1)$ as $b \rightarrow b_{H}$, where $Z_{0}$ is a $N(0,1)$ random variable. It follows that $J_{2}(b)=h_{n}(b)$.

To prove $J_{3}(b)=h_{n}(b)$, note that the difference $W_{i}-W_{i}(b)$, as given in (3.6) and (3.3) equals to

$$
\frac{\bar{\xi}_{b}\left(Z_{i}\right) \delta_{i}}{\bar{G}\left(Z_{i}\right)}-\int \bar{\xi}_{b}(x) \mathrm{d} F(x)+\frac{\bar{\delta}_{i}}{\bar{H}\left(Z_{i}\right)} \bar{\psi}_{b}\left(Z_{i}\right)-\int \bar{\psi}_{b}(s) \frac{I\left[Z_{i} \geq s\right]}{\bar{H}^{2}(s)} d H^{0}(s) .
$$

Therefore $W_{i}-W_{i}(b)$ are i.i.d. random variables with mean zero and variance $\bar{\sigma}_{b}^{2}$. Hence, $J_{3}(b)=h_{n}(b)$ follows for the same reason as that of $J_{2}(b)$.

We conclude that the following holds for (3.13),

$$
\sqrt{n} \int \xi(x) \mathrm{d}\left(F_{n}(x)-F(x)\right)-\frac{1}{\sqrt{n}} \sum_{i=1}^{n} W_{i}=o_{p}(1)+h_{n}(b)+h_{n}(b) .
$$

Theorem 3.1 follows from Lemma 3.2.

Remark 3.3 If $b_{F}<b_{G}$, then (1.3) is equivalent to $\xi$ having finite second moment. If $\xi$ is bounded and away from zero, then (1.3) is equivalent to $\int_{0}^{\infty} d F(s) / \bar{G}(s)$. 


\section{Empirical Likelihood Ratios and Confidence In- tervals for $\theta$}

To develop an EL inference procedure, we consider a specific $g(x, \theta)$. For each fixed $\theta$, as before, set $\xi(x)=g(x, \theta)$. We shall utilize the i.i.d. random variables

$$
W_{i}=\frac{\xi\left(Z_{i}\right) \delta_{i}}{\bar{G}\left(Z_{i}\right)}+\frac{\bar{\delta}_{i}}{\bar{H}\left(Z_{i}\right)} \psi\left(Z_{i}\right)-\int \psi(s) \frac{I\left[Z_{i} \geq s\right]}{\bar{H}^{2}(s)} d H^{0}(s) .
$$

to obtain an estimating equation for the EL ratio. Recall that $\mu=\mathrm{E} W_{i}=\int_{0}^{\infty} \xi(x) \mathrm{d} F(x)$ and $\operatorname{Var}\left(W_{i}\right)$ are given by (3.10). Note that setting $\xi(x)=g(x, \theta)$ above has nothing to do with defining $\theta$ from the equation $\operatorname{Eg}(X, \theta)=0$ as given in (1.5). If, however, the true parameter $\theta_{0}$ is the solution of the equation

$$
\mathrm{E} g(Y, \theta)=\int g(x, \theta) \mathrm{d} F(x)=0,
$$

then $\xi(x)=g\left(x, \theta_{0}\right)$ is such that

$$
\mu=\mathrm{E} W_{i}=\int_{0}^{\infty} \xi(x) \mathrm{d} F(x)=0 .
$$

Regarding $W_{i}$ for $i=1, \cdots, n$ as a "complete" random sample, one could formulate an EL likelihood ratio $R\left(\theta_{0}\right)$ with multinomial probability $p_{i}$ assigned to $W_{i}$ and the constraint $\sum_{i=1}^{n} W_{i} p_{i}=0$. However, $W_{i}^{\prime} s$ are not observable because of the unknown distributions $G, F H$ and $H^{0}$. We shall replace them by the KM estimates, $F_{n}, G_{n}$ given by (2.3) and an estimate of $\psi$,

$$
\psi_{n}(x)=\int_{s \geq x} \xi(s) \mathrm{d} F_{n}(s) .
$$

Replacing $\bar{G}, \bar{H}, H^{0}$ in (4.1) by their corresponding empirical distributions in (2.2) gives an approximation of $W_{i}$ in (4.1) by

$$
W_{n i}=\frac{\xi\left(Z_{i}\right) \delta_{i}}{\bar{G}_{n}\left(Z_{i}-\right)}+\frac{\bar{\delta}_{i}}{\bar{H}_{n}\left(Z_{i}-\right)} \psi_{n}\left(Z_{i}\right)-\int \psi_{n}(s) \frac{I\left[Z_{i} \geq s\right]}{\bar{H}_{n}^{2}(s-)} \mathrm{d} H_{n}^{0}(s) .
$$

The price to pay for the estimation is that $W_{n i}{ }^{\prime} s$ are not stochastically independent which complicates the ensuing analysis. 
The following theorem indicates the possibility of using $W_{n i}$ to construct empirical likelihood ratio and to obtain asymptotically a standard $\chi^{2}$ distribution.

Theorem 4.1. Let $W_{n i}$ be given by (4.4) and $E \xi(Y)=0$. Then under condition (1.3), as $n \rightarrow \infty$, we have

$$
\frac{1}{\sqrt{n}} \sum_{i=1}^{n} W_{n i}=\frac{1}{\sqrt{n}} \sum_{i=1}^{n} W_{i}+o_{p}(1)
$$

Proof. By (2.6), we have

$$
\begin{aligned}
& \frac{1}{n} \sum_{i=1}^{n} W_{n i} \\
= & \frac{1}{n} \sum_{i=1}^{n}\left(\frac{\xi\left(Z_{i}\right) \delta_{i}}{\bar{G}_{n}\left(Z_{i}-\right)}+\frac{\bar{\delta}_{i}}{\bar{H}_{n}\left(Z_{i}-\right)} \psi_{n}\left(Z_{i}\right)-\int \psi_{n}(s) \frac{I\left[Z_{i} \geq s\right]}{\bar{H}_{n}^{2}(s-)} d H_{n}^{0}(s)\right) \\
= & \int \frac{\xi(s)}{\bar{G}_{n}(s-)} \mathrm{d} H_{n}^{1}(s)+\int \frac{\psi_{n}(s)}{\bar{H}_{n}(s-)} \mathrm{d} H_{n}^{0}(s)-\int \psi_{n}(s) \frac{\bar{H}_{n}(s-)}{\bar{H}_{n}^{2}(s-)} \mathrm{d} H_{n}^{0}(s) \\
= & \int \frac{\xi(s)}{\bar{G}_{n}(s-)} \mathrm{d} H_{n}^{1}(s)=\int \xi(s) \mathrm{d} F_{n}(s) .
\end{aligned}
$$

Applying $\int_{0}^{\infty} \xi(s) \mathrm{d} F(s)=\mathrm{E} \xi(Y)=0$ and Theorem 3.1, we arrive at

$$
\frac{1}{\sqrt{n}} \sum_{i=1}^{n} W_{n i}=\sqrt{n} \int \xi(s) \mathrm{d}\left(F_{n}(s)-F(s)\right)=\frac{1}{\sqrt{n}} \sum_{i=1}^{n} W_{i}+o_{p}(1) .
$$

Following Owen (2001), define the EL ratio of $\theta$ by a multinomial likelihood subject to constraints as

$$
R(\theta)=\sup _{\left\{p_{i}\right\}}\left\{\prod_{i=1}^{n} n p_{i} \mid \sum_{i=1}^{n} p_{i}=1, \sum_{i=1}^{n} p_{i} W_{n i}=0, p_{i} \geq 0, i=1,2, \cdots, n\right\} .
$$

To determine $R(\theta)$, we solve, as usual, for the Lagrange multipliers $\mu$ and $\lambda$ in

$$
A=\sum_{i=1}^{n} \log \left(n p_{i}\right)-n \lambda\left(\sum_{i=1}^{n} p_{i} W_{n i}\right)-\mu\left(1-\sum_{i=1}^{n} p_{i}\right)
$$

Then $\mu=-n$ and $p_{i}=\frac{1}{n}\left(1+\lambda W_{n i}\right)^{-1}, i=1,2, \cdots, n$, where $\lambda$ is the solution of

$$
\frac{1}{n} \sum_{i=1}^{n} \frac{W_{n i}}{1+\lambda W_{n i}}=0
$$


The uniqueness of $\lambda$ will be addressed in the proof of Theorem 4.2. The EL ratio of $\theta$ can be written as

$$
R(\theta)=\prod_{i=1}^{n}\left(n p_{i}\right)=\prod_{i=1}^{n}\left(1+\lambda W_{n i}\right)^{-1} .
$$

Theorem 4.2. Suppose that $\theta_{0}$ is the unique solution of (4.2) and finite second moment (1.3) holds. Set $\xi(x)=g\left(x, \theta_{0}\right)$. Then $l\left(\theta_{0}\right)=-2 \log R\left(\theta_{0}\right)$ converges in distribution to a $\chi_{1}^{2}$ random variable with one degree of freedom, as $n \rightarrow \infty$.

Applying Theorem 4.2, confidence intervals for $\theta$ can be constructed as

$$
I_{1}=\left\{\theta: l(\theta) \leq c_{1-\alpha}\right\}
$$

where $c_{1-\alpha}$ is the $(1-\alpha)$ th quantile of the $\chi_{1}^{2}$ distribution. $I_{1}$ has asymptotic coverage probability of $1-\alpha$, as $n \rightarrow \infty$.

To prove Theorem 4.2, we shall make use of the following Taylor's expansion of $-2 \log R\left(\theta_{0}\right)$,

$$
\begin{aligned}
-2 \log R\left(\theta_{0}\right) & =2 \sum_{i=1}^{n} \ln \left(1+\lambda_{n} W_{n i}\right) \\
& =\sum_{i=1}^{n} 2\left(\lambda_{n} W_{n i}-\frac{1}{2} \lambda_{n}^{2} W_{n i}^{2}+\eta_{i}\right) \quad\left(\text { here }\left|\eta_{i}\right| \leq\left|X_{i}\right|^{3}\right) \\
& =2 \lambda_{n} n \bar{W}_{n}-\lambda_{n}^{2} n S_{n}^{2}+2 \sum_{i=1}^{n} \eta_{i} \\
& =2 n \frac{\bar{W}_{n}^{2}}{\sigma^{2}}-\frac{1}{\sigma^{4}} n \bar{W}_{n}^{2}\left(\sigma^{2}+o_{p}(1)\right)+o_{p}(1) \\
& =\frac{n \bar{W}_{n}^{2}}{\sigma^{2}}+o_{p}(1) \rightarrow \chi_{1}^{2}, \quad \text { in distribution, }
\end{aligned}
$$

where

$$
\bar{W}_{n}=\sum_{i=1}^{n} W_{n i}, \quad \text { and } \quad S_{n}^{2}=\sum_{i=1}^{n} W_{n i}^{2} .
$$

Asymptotic analysis of $\bar{W}_{n}=\sum_{i=1}^{n} W_{n i}$ and $S_{n}^{2}=\sum_{i=1}^{n} W_{n i}^{2}$ are needed for establishing the last two equalities in the expansion. It will be proven in Lemma 4.3 and Theorem 
4.2 that both of these averages are related to the asymptotic variance (1.4) or (3.10). The following lemmas are needed for proving Theorem 4.2.

Lemma 4.1 Let $f_{n}(x)$ and $f(x)$ be monotone functions defined on the range of $Z$. If $f(x)$ is continuous and for $x \in\left[0, b_{H}\right], f_{n}(x) \rightarrow f(x)$, as $n \rightarrow \infty$, then $f_{n}(x)$ converges to $f(x)$ uniformly on $\left[0, b_{H}\right]$. The proof is omitted.

Lemma 4.2 Let $V_{n i}=W_{n i} \bar{H}_{n}\left(Z_{i}-\right) \bar{H}\left(Z_{i}\right)$ and $V_{i}=W_{i} \bar{H}^{2}\left(Z_{i}\right)$. Under the conditions of Theorem 4.2 and $\theta=\theta_{0}$, as $n \rightarrow \infty$,
(1) $\frac{1}{n} \sum_{i=1}^{n}\left(W_{n i}-W_{i}\right)^{2}=o_{p}(1)$,
(2) $\frac{1}{n} \sum_{i=1}^{n}\left(V_{n i}-V_{i}\right)^{2} \rightarrow 0$, a.s..

The proof is relegated to the Appendix. Lemma 4.2 is needed for showing that with probability 1 , for large $n$ the set $\left\{W_{n i}\right\}$ contains a positive and a negative value. To facilitate the proof, $V_{n i}^{\prime} s$, a modification of $W_{n i}^{\prime} s$, are introduced to deal with the problem at the boundary $b_{H}$. It follows that for large $n$ there exists a unique $\lambda_{n}$ for $R(\theta)$ in $(4.8)$.

Lemma 4.3 Let $W_{n i}$ and $W_{i}$ be given by (4.4) and (4.1), respectively. Under the conditions of Theorem 4.2 and $\theta=\theta_{0}$, as $n \rightarrow \infty$,

$$
\max _{1 \leq i \leq n}\left|W_{n i}\right|=o_{p}(\sqrt{n}), \quad \frac{1}{n} \sum_{i=1}^{n} W_{n i}^{2}=\sigma^{2}+o_{p}(1),
$$

and

$$
\frac{1}{\sqrt{n}} \sum_{i=1}^{n} W_{n i} \rightarrow N\left(0, \sigma^{2}\right), \quad \text { in dist. }
$$

where $\sigma^{2}=\operatorname{Var}\left(W_{i}\right)$ is given by (3.10).

The proof is relegated to the Appendix. We now prove Theorem 4.2.

Proof of Theorem 4.2. The Lagrange multiplier $\lambda$ in (4.6) appears in the equation

$$
h(\lambda)=\frac{1}{n} \sum_{i=1}^{n} \frac{W_{n i}}{1+\lambda W_{n i}}=0 .
$$


We shall show that for large $n, h(\lambda)=0$ has a unique solution $\lambda_{n}$ such that $\lambda_{n} W_{n i}>-1$ for all $i$. Put

$$
U_{i}= \begin{cases}-W_{n i}^{-1}, & W_{n i} \neq 0 \\ \infty, & W_{n i}=0\end{cases}
$$

Let $U_{(1)} \leq U_{(2)} \leq \cdots \leq U_{(n)}$ be the ordered statistics of $U_{1}, U_{2}, \cdots, U_{n}$. Then

$$
h(\lambda)=\frac{1}{n} \sum_{i=1}^{n} \frac{W_{n i}}{1+\lambda W_{n i}}=\frac{1}{n} \sum_{i=1}^{n} \frac{1}{\lambda-U_{(i)}}
$$

is monotone and differentiable in $\lambda$ on each nonempty interval $\left(U_{(i)}, U_{(i+1)}\right)$. We claim that for large $n$, there exists an $i$ such that $U_{(i)}<0<U_{(i+1)}$. To see this, we note that for every $\varepsilon>0$, by Lemma $4.2(2)$

$$
\frac{1}{n} \sum_{i=1}^{n} I\left[\left|V_{n i}-V_{i}\right| \geq \varepsilon\right] \leq \frac{1}{n} \sum_{i=1}^{n}\left(V_{n i}-V_{i}\right)^{2} / \varepsilon^{2}=o(1), \text { a.s.. }
$$

Using the fact that $I\left[V_{i} \geq \varepsilon\right] \leq I\left[V_{n i} \geq \varepsilon / 2\right]+I\left[\left|V_{n i}-V_{i}\right| \geq \varepsilon / 2\right]$, we get

$$
\frac{1}{n} \sum_{i=1}^{n} I\left[V_{n i} \geq \varepsilon / 2\right] \geq \frac{1}{n} \sum_{i=1}^{n} I\left[V_{i} \geq \varepsilon\right]+o(1), \text { a.s.. }
$$

Using the fact that $P\left(V_{1}>0\right)=P\left(W_{1}>0\right)>0$, it is seen that for some $\varepsilon>0$,

$$
\liminf _{n \rightarrow \infty} \frac{1}{n} \sum_{i=1}^{n} I\left[V_{n i} \geq \varepsilon / 2\right] \geq P\left(V_{1} \geq \varepsilon\right)>0, \text { a.s.. }
$$

Similarly, we have

$$
\liminf _{n \rightarrow \infty} \frac{1}{n} \sum_{i=1}^{n} I\left[V_{n i} \leq-\varepsilon / 2\right] \geq P\left(V_{1} \leq-\varepsilon\right)>0, \text { a.s.. }
$$

Since $W_{n i}$ and $V_{n i}$ have the same sign, hence the claim is true. It follows that there is a unique $\lambda_{n} \in\left(U_{(i)}, U_{(i+1)}\right)=\left(-1 / \max \left\{W_{n i}\right\},-1 / \min \left\{W_{n i}\right\}\right)$ such that $h\left(\lambda_{n}\right)=0$ and $\lambda_{n} \max \left\{W_{n i}\right\}>-1$ and $\lambda_{n} \min \left\{W_{n i}\right\}>-1$.

The rest of the proof is similar to that of Owen (2001). In fact, setting

$$
X_{i}=\lambda_{n} W_{n i}, \quad \bar{W}_{n}=\frac{1}{n} \sum_{i=1}^{n} W_{n i}, \quad S_{n}^{2}=\frac{1}{n} \sum_{i=1}^{n} W_{n i}^{2},
$$


we have $S_{n}^{2}=\sigma^{2}+o_{p}(1)$,

$$
\frac{1}{n} \sum_{i=1}^{n} \frac{X_{i}^{2}}{1+X_{i}}=\frac{1}{n} \sum_{i=1}^{n} \frac{\left(X_{i}+1-1\right) X_{i}}{1+X_{i}}=\bar{X}_{n}-\lambda_{n} h\left(\lambda_{n}\right)=\lambda_{n} \bar{W}_{n}
$$

and

$$
\lambda_{n}^{2} S_{n}^{2}=\frac{1}{n} \sum_{i=1}^{n} X_{i}^{2} \leq \frac{1}{n} \sum_{i=1}^{n} \frac{X_{i}^{2}}{1+X_{i}}\left(1+\max _{1 \leq j \leq n}\left|X_{j}\right|\right)=\lambda_{n} \bar{W}_{n}+\lambda_{n}^{2} o_{p}(1) .
$$

It follows that

$$
\lambda_{n}=\frac{\bar{W}_{n}}{\sigma^{2}+o_{p}(1)}=O_{p}\left(n^{-1 / 2}\right)
$$

and

$$
\bar{W}_{n}=\lambda_{n} \sigma^{2}+o_{p}\left(n^{-1 / 2}\right) .
$$

Applying Lemma 4.3, we have

$$
\sum_{i=1}^{n}\left|X_{i}\right|^{3} \leq \lambda_{n}^{3} \sum_{i=1}^{n}\left|W_{n i}\right|^{2} \max _{1 \leq j \leq n}\left|W_{n i}\right|=O_{p}\left(n^{-3 / 2}\right) O_{p}(n) o_{p}\left(n^{1 / 2}\right)=o_{p}(1) .
$$

Therefore the Taylor expansion (above (4.10)) is valid from which the theorem follows.

Remark 4.1 We are able to obtain the standard asymptotic $\chi_{1}^{2}$ distribution for $-2 \log R\left(\theta_{0}\right)$ is because the asymptotic variance of

$$
\frac{1}{\sqrt{n}} \sum_{i=1}^{n} W_{n i}=\frac{1}{\sqrt{n}} \sum_{i=1}^{n} W_{i}+o_{p}(1)
$$

(which is $\sigma^{2}=\operatorname{Var}\left(W_{i}\right)$ ) equals the limit of (see Lemma 4.3)

$$
\frac{1}{n} \sum_{i=1}^{n} W_{n i}^{2}=\frac{1}{n} \sum_{i=1}^{n} W_{i}^{2}+o_{p}(1)
$$

If $-2 \log (E L$ ratio $)$ is based on the estimating function $M_{2}=M_{2}(Z, \delta, \theta)$ in (3.8) ( or in (3.7)), then

$$
V_{n i}=\frac{g\left(Z_{i}, \theta\right) \delta_{i}}{1-G_{n}\left(Z_{i}\right)}
$$


will be used to construct $-2 \log ($ EL ratio $)$. Now, the asymptotic variance of

$$
\frac{1}{\sqrt{n}} \sum_{i=1}^{n} V_{n i}=\frac{1}{\sqrt{n}} \sum_{i=1}^{n} W_{i}+o_{p}(1)
$$

is $\sigma^{2}$ (see(3.10)), but the limit of $\frac{1}{n} \sum_{i=1}^{n} V_{n i}^{2}$ or

$$
\frac{1}{n} \sum_{i=1}^{n}\left(V_{n i}-\frac{1}{n} \sum_{j=1}^{n} V_{n j}\right)^{2}
$$

is (see Remark 3.1)

$$
\sigma_{1}^{2}=\int \frac{\xi^{2}(s)}{\bar{G}(s)} d F(s)-\mu^{2}, \quad \mu=0 .
$$

Therefore, a scaled parameter $r=\sigma_{1}^{2} / \sigma^{2}$ must be introduced in order to obtain the asymptotic distribution for $-2 \log ($ EL ratio) as in Wang \& Jing (2001).

\section{Simulation}

Simulations are carried out to study and compare finite sample performance of confidence intervals $I_{1}$ in (4.9) derived from Theorem 4.2 and $I_{2}$ from the scaled $\chi_{1}^{2}$ distribution given by Wang \& Jing (2001) and Qin \& Zhao (2007).

To calculate $I_{1}, W_{n i}$ in (4.4) is used, where

$$
W_{n i}=\frac{\xi\left(Z_{i}\right) \delta_{i}}{\bar{G}_{n}\left(Z_{i}-\right)}+\frac{\bar{\delta}_{i}}{\bar{H}_{n}\left(Z_{i}-\right)} \psi_{n}\left(Z_{i}\right)-\frac{1}{n} \sum_{j=1}^{n} \psi_{n}\left(Z_{j}\right) \frac{I\left[Z_{i} \geq Z_{j}\right] \bar{\delta}_{j}}{\bar{H}_{n}^{2}\left(Z_{j}-\right)},
$$

and $\psi_{n}(x)$ is given by (4.3).

Confidence intervals $I_{2}$ are calculated as follows. Let $F_{n}$, and $G_{n}$ be the Kaplan-Meier estimators defined by (2.3). Suppose $\hat{\theta}$ is the unique solution of $\int g(s, \theta) \mathrm{d} F_{n}(s)=0$. Set

$$
\begin{gathered}
\xi_{i}=g\left(Z_{i}, \theta\right), \quad \hat{\xi}_{i}=g\left(Z_{i}, \hat{\theta}\right), \\
V_{n i}=\frac{\xi_{i} \delta_{i}}{1-G_{n}\left(Z_{i}\right)}, \quad \hat{V}_{n i}=\frac{\hat{\xi}_{i} \delta_{i}}{1-G_{n}\left(Z_{i}\right)}, \\
\sigma_{1}^{2}=\frac{1}{n} \sum_{i=1}^{n}\left(\hat{V}_{n i}-\bar{V}_{n}\right)^{2}, \quad \bar{V}_{n}=\frac{1}{n} \sum_{i=1}^{n} \hat{V}_{n i},
\end{gathered}
$$




$$
\hat{r}=\frac{\sigma_{1}^{2}}{n \widehat{\operatorname{Var}}^{*}(j a c k)},
$$

where $n \widehat{\operatorname{Var}}^{*}(j a c k)$ is the modified jackknife estimator of the asymptotic variance of $\hat{\xi}$ given in Stute(1996). Then, the EL-based confidence interval for $\theta$ is

$$
I_{2}=\left\{\theta: 2 \hat{r} \sum_{i=1}^{n} \log \left(1+\lambda V_{n i}\right) \leq c_{1-\alpha}\right\},
$$

where $\lambda$ is the solution of $\sum_{i=1}^{n} V_{n i} /\left(1+\lambda V_{n i}\right)=0$.

Simulations were performed in two scenarios. In scenario I, the parameter of interest is $\theta_{0}=\mathrm{E} Y$ and in scenario II, the mean residual lifetime.

Scenario I: The parameter of interest is $\theta_{0}=\operatorname{E} Y$ and $\xi(x)=g(x, \theta)=x-\theta$ is used for calculating $I_{1}$. Two cases were simulated:

(i) The lifetime $Y$ is uniformly distributed on $(0,1)$ and the censoring time $C$ is uniformly distributed on $(0, c)$. We selected $c=2.5$ and $c=1.3$ which corresponds respectively to $20 \%$ and $30 \%$ censoring proportions.

(ii) $Y$ has a Weibull $(1,10)$ distribution and $C$ has an $\operatorname{Exp}(\lambda)$ distribution. Then for $\lambda=4.3$ and $\lambda=2.7$, the corresponding censoring proportions are $20 \%$ and $30 \%$. The simulated observations are $n$ i.i.d. copies of $Z=\min (Y, C), \delta=I[Y \leq C]$. Based on the simulated observations, confidence intervals $I_{1}$ derived from Theorem 4.2 and $I_{2}$ from (5.2) were calculated. The process was repeated for $N=2 \times 10^{4}$ times and the coverage proportions and the average width of the confidence intervals were calculated using the $N$ data sets. The results are summarized in Table 1 and Table 2.

The following are noted.

(1) As the sample size $n$ increases, all of the coverage proportions converge to the nominal level $1-\alpha$.

(2) For Uniform $(0,1)$ distribution, $I_{1}$ has better coverage proportions. In 8/16 of the cases, the average width of $I_{2}$ is slightly shorter than that of $I_{1}$. In 8/16 of the cases, $I_{2}$ and $I_{1}$ have the same average width. 
Table 1: The coverage proportions for the true $\theta_{0}=\mathrm{E} Y$

\begin{tabular}{|c|c|c|c|c|c|}
\hline \multicolumn{6}{|c|}{$20 \%$ censoring proportion } \\
\hline \multirow{2}{*}{$\begin{array}{c}\text { Nominal Value } \\
1-\alpha\end{array}$} & \multirow{2}{*}{$\begin{array}{c}\text { Sample Size } \\
n\end{array}$} & \multicolumn{2}{|c|}{ Uniform $(0,1)$} & \multicolumn{2}{|c|}{ Weibull $(1,10)$} \\
\hline & & $I_{2}$ & $I_{1}$ & $I_{2}$ & $I_{1}$ \\
\hline \multirow[t]{4}{*}{0.90} & 20 & 0.876 & 0.881 & 0.871 & 0.871 \\
\hline & 40 & 0.895 & 0.897 & 0.889 & 0.890 \\
\hline & 60 & 0.897 & 0.897 & 0.893 & 0.893 \\
\hline & 80 & 0.897 & 0.898 & 0.896 & 0.896 \\
\hline \multirow[t]{4}{*}{0.95} & 20 & 0.928 & 0.935 & 0.922 & 0.924 \\
\hline & 40 & 0.946 & 0.949 & 0.939 & 0.941 \\
\hline & 60 & 0.947 & 0.948 & 0.945 & 0.946 \\
\hline & 80 & 0.947 & 0.947 & 0.947 & 0.948 \\
\hline \multicolumn{6}{|c|}{$30 \%$ censoring proportion } \\
\hline \multirow{2}{*}{$\begin{array}{c}\text { Nominal Value } \\
1-\alpha\end{array}$} & \multirow{2}{*}{$\begin{array}{c}\text { Sample Size } \\
n\end{array}$} & \multicolumn{2}{|c|}{ Uniform $(0,1)$} & \multicolumn{2}{|c|}{ Weibull $(1,10)$} \\
\hline & & $I_{2}$ & $I_{1}$ & $I_{2}$ & $I_{1}$ \\
\hline \multirow[t]{4}{*}{0.90} & 20 & 0.841 & 0.861 & 0.867 & 0.869 \\
\hline & 40 & 0.885 & 0.890 & 0.890 & 0.891 \\
\hline & 60 & 0.888 & 0.892 & 0.890 & 0.891 \\
\hline & 80 & 0.897 & 0.900 & 0.893 & 0.894 \\
\hline \multirow[t]{4}{*}{0.95} & 20 & 0.897 & 0.916 & 0.916 & 0.924 \\
\hline & 40 & 0.934 & 0.941 & 0.939 & 0.943 \\
\hline & 60 & 0.941 & 0.946 & 0.944 & 0.946 \\
\hline & 80 & 0.945 & 0.947 & 0.945 & 0.947 \\
\hline
\end{tabular}


Table 2: The average width of confidence intervals for $\theta_{0}=\mathrm{E} Y$

\begin{tabular}{cccccc}
\hline $20 \%$ censoring proportion & \multicolumn{2}{c}{ width } & \multicolumn{2}{c}{ width } \\
\hline Nominal Value & Sample Size & \multicolumn{2}{c}{ Uniform $(0,1)$} & \multicolumn{2}{c}{ Weibull $(1,10)$} \\
$1-\alpha$ & $n$ & $I_{2}$ & $I_{1}$ & $I_{2}$ & $I_{1}$ \\
\hline 0.90 & 20 & 0.217 & 0.218 & 0.092 & 0.091 \\
& 40 & 0.157 & 0.157 & 0.066 & 0.065 \\
& 60 & 0.129 & 0.129 & 0.054 & 0.053 \\
& 80 & 0.112 & 0.112 & 0.046 & 0.046 \\
\hline 0.95 & 20 & 0.258 & 0.259 & 0.110 & 0.109 \\
& 40 & 0.187 & 0.187 & 0.079 & 0.078 \\
& 60 & 0.154 & 0.154 & 0.064 & 0.064 \\
$30 \%$ censoring proportion & \multicolumn{2}{c}{ width } & \multicolumn{2}{c}{ width } \\
\hline Nominal Value & Sample Size & Uniform $(0,1)$ & Weibull $(1,10)$ \\
$1-\alpha$ & $n$ & $I_{2}$ & $I_{1}$ & $I_{2}$ & $I_{1}$ \\
\hline 0.90 & 20 & 0.220 & 0.227 & 0.097 & 0.096 \\
& 40 & 0.162 & 0.164 & 0.069 & 0.069 \\
& 60 & 0.134 & 0.134 & 0.057 & 0.057 \\
& 80 & 0.116 & 0.116 & 0.049 & 0.049 \\
\hline 0.95 & 20 & 0.260 & 0.270 & 0.116 & 0.116 \\
& 40 & 0.192 & 0.196 & 0.083 & 0.083 \\
& 60 & 0.159 & 0.160 & 0.068 & 0.068 \\
& 80 & 0.138 & 0.139 & 0.059 & 0.059 \\
\hline
\end{tabular}


Table 3: The sample variances of $\left\{W_{n i}\right\}$ and $\left\{V_{n i}\right\}, \theta_{0}=\mathrm{E} Y$

\begin{tabular}{|c|c|c|c|c|}
\hline \multicolumn{5}{|c|}{$20 \%$ censoring proportion } \\
\hline & \multicolumn{2}{|c|}{ Uniform $(0,1)$} & \multicolumn{2}{|c|}{ Weibull $(1,10)$} \\
\hline Sample Size $n$ & $s_{W}^{2}$ & $s_{V}^{2}$ & $s_{W}^{2}$ & $s_{V}^{2}$ \\
\hline 20 & 0.0935 & 0.1121 & 0.0157 & 0.0163 \\
\hline 40 & 0.0938 & 0.1115 & 0.0157 & 0.0162 \\
\hline 60 & 0.0937 & 0.1107 & 0.0157 & 0.0161 \\
\hline 80 & 0.0934 & 0.1100 & 0.0158 & 0.0162 \\
\hline \multicolumn{5}{|c|}{$30 \%$ censoring proportion } \\
\hline & \multicolumn{2}{|c|}{ Uniform $(0,1)$} & \multicolumn{2}{|c|}{ Weibull $(1,10)$} \\
\hline Sample Size $n$ & $s_{W}^{2}$ & $s_{V}^{2}$ & $s_{W}^{2}$ & $s_{V}^{2}$ \\
\hline 20 & 0.1005 & 0.1386 & 0.0175 & 0.0185 \\
\hline 40 & 0.1013 & 0.1401 & 0.0176 & 0.0184 \\
\hline 60 & 0.1016 & 0.1402 & 0.0176 & 0.0183 \\
\hline 80 & 0.1012 & 0.1393 & 0.0176 & 0.0183 \\
\hline
\end{tabular}

(3) For Weibull $(1,10)$ distribution, $I_{1}$ has better coverage proportion and width.

In the $j$ th simulation, $\left\{W_{n i}\right\}$ and $\left\{V_{n i}\right\}$ were calculated according to (4.4) and (5.1) respectively. Then the sample means of $\left\{W_{n i}\right\}$ and $\left\{V_{n i}\right\}$ are the same (see the proof of Theorem 4.1). But the sample variance $s_{W}^{2}(j)$ of $\left\{W_{n i}\right\}$ and the sample variance $s_{V}^{2}(j)$ of $\left\{V_{n i}\right\}$ are different. Let

$$
s_{W}^{2}=\frac{1}{N} \sum_{j=1}^{N} s_{W}^{2}(j), \quad s_{V}^{2}=\frac{1}{N} \sum_{j=1}^{N} s_{V}^{2}(j) .
$$

Table 3 shows that the sample variance of $\left\{W_{n i}\right\}$ is smaller than that of $\left\{V_{n i}\right\}$. This is proved in Remark 3.1 for the population variances.

Scenario II: Let $g(x, \theta)=\left(x-t_{0}-\theta\right) I\left[x \geq t_{0}\right]$. Then by solving the equation E $g(Y, \theta)=0$, we obtain the mean residual life of $Y$,

$$
\theta_{0}=\mathrm{E}\left(Y-t_{0} \mid Y \geq t_{0}\right)=\frac{\mathrm{E}\left(Y-t_{0}\right) I\left[Y \geq t_{0}\right]}{P\left(Y \geq t_{0}\right)}
$$

as studied in Qin \& Zhao (2007). Let $Y$ have a Weibull $(1,10)$ distribution and $C$ have an $\operatorname{Exp}(\lambda)$ distribution. By setting $\lambda=4.3$ and $\lambda=2.7$, we achieved $20 \%$ and $30 \%$ censoring proportions respectively. As in Scenario I, each simulation was repeated 
Table 4: The coverage proportion and average width of confidence intervals for $\theta_{0}=\mathrm{E}\left(Y-t_{0} \mid Y \geq t_{0}\right)$ under the assumptions of $Y \sim$ Weibull(1,10), 20\% censoring proportion, and $1-\alpha=0.90$.

\begin{tabular}{cccccccccc}
\hline Sample Size $n$ & Method & \multicolumn{4}{c}{ Coverage Ratio } & \multicolumn{4}{c}{ Average Width } \\
\hline & & \multicolumn{4}{c}{$P\left(Y \geq t_{0}\right)$} & \multicolumn{4}{c}{$P\left(Y \geq t_{0}\right)$} \\
& & 0.90 & 0.70 & 0.50 & 0.30 & 0.90 & 0.70 & 0.50 & 0.30 \\
\hline 20 & $I_{2}$ & 0.878 & 0.851 & 0.795 & 0.659 & 0.074 & 0.062 & 0.054 & 0.044 \\
& $I_{1}$ & 0.881 & 0.863 & 0.820 & 0.701 & 0.074 & 0.062 & 0.056 & 0.048 \\
\hline 40 & $I_{2}$ & 0.889 & 0.878 & 0.859 & 0.800 & 0.053 & 0.046 & 0.042 & 0.039 \\
& $I_{1}$ & 0.891 & 0.884 & 0.874 & 0.833 & 0.053 & 0.046 & 0.043 & 0.041 \\
\hline 60 & $I_{2}$ & 0.897 & 0.892 & 0.877 & 0.839 & 0.044 & 0.037 & 0.035 & 0.034 \\
& $I_{1}$ & 0.898 & 0.897 & 0.888 & 0.863 & 0.044 & 0.038 & 0.035 & 0.035 \\
\hline 80 & $I_{2}$ & 0.895 & 0.888 & 0.884 & 0.853 & 0.038 & 0.033 & 0.031 & 0.030 \\
& $I_{1}$ & 0.896 & 0.892 & 0.892 & 0.871 & 0.038 & 0.033 & 0.031 & 0.031 \\
\hline
\end{tabular}

$N=2 \times 10^{4}$ times. The coverage proportion of the $N$ data sets and their average width were calculated. The results are summarized in Table 4 and Table 5, respectively.

The following are noted from Tables 4 and 5 .

(1) As the sample size $n$ increases, all of the coverage proportions increase and are close to the nominal levels.

(2) The coverage proportions of $I_{1}$ are much better than that of $I_{2}$.

(3) In 15/32 of the cases, the average width of $I_{2}$ is slightly shorter than that of $I_{1}$. In $17 / 32$ of the cases, $I_{2}$ and $I_{1}$ have the same average width.

\section{Appendix}

Proof of Lemma 3.2. Put $\eta_{n}=o_{p}(1)$. By assumptions, for any $\varepsilon>0$ and $\delta>0$, there exist $M>0, b<b_{H}$ and $n_{0}>1$ such that for $n \geq n_{0}, P\left(\left|V_{n}\right| \geq M\right) \leq \delta$, $P\left(\left|h_{n}(b)\right| \geq \varepsilon / M\right) \leq P(|h(b)| \geq \varepsilon / M)+\delta / 2 \leq \delta$ and $P\left(\left|\eta_{n}\right| \geq \varepsilon\right)<\delta$. It follows that 
Table 5: The coverage ratio and average width of confidence intervals for $\theta_{0}=\mathrm{E}(Y-$ $\left.t_{0} \mid Y \geq t_{0}\right)$, under the assumptions $Y \sim$ Weibull $(1,10), 30 \%$ censoring proportion and $1-\alpha=0.90$.

\begin{tabular}{cccccccccc}
\hline Sample Size $n$ & Method & \multicolumn{4}{c}{ Coverage Ratio } & \multicolumn{4}{c}{ Average Width } \\
\hline & & \multicolumn{4}{c}{$P\left(Y \geq t_{0}\right)$} & & \multicolumn{4}{c}{$P\left(Y \geq t_{0}\right)$} \\
& & 0.90 & 0.70 & 0.50 & 0.30 & 0.90 & 0.70 & 0.50 & 0.30 \\
\hline \multirow{2}{*}{20} & $I_{2}$ & 0.864 & 0.833 & 0.760 & 0.605 & 0.079 & 0.065 & 0.055 & 0.043 \\
& $I_{1}$ & 0.872 & 0.851 & 0.793 & 0.659 & 0.079 & 0.065 & 0.058 & 0.048 \\
\hline \multirow{2}{*}{40} & $I_{2}$ & 0.887 & 0.872 & 0.846 & 0.777 & 0.057 & 0.048 & 0.045 & 0.041 \\
& $I_{1}$ & 0.891 & 0.882 & 0.867 & 0.818 & 0.057 & 0.049 & 0.046 & 0.043 \\
\hline 60 & $I_{2}$ & 0.892 & 0.888 & 0.870 & 0.822 & 0.046 & 0.040 & 0.037 & 0.036 \\
& $I_{1}$ & 0.895 & 0.895 & 0.884 & 0.851 & 0.046 & 0.040 & 0.038 & 0.037 \\
\hline 80 & $I_{2}$ & 0.892 & 0.888 & 0.878 & 0.845 & 0.040 & 0.035 & 0.033 & 0.032 \\
& $I_{1}$ & 0.895 & 0.895 & 0.887 & 0.869 & 0.040 & 0.035 & 0.033 & 0.033 \\
\hline
\end{tabular}

for $n \geq n_{0}$

$$
\begin{aligned}
P\left(\left|S_{n}\right| \geq 2 \varepsilon\right) & \leq P\left(\left|\eta_{n}\right| \geq \varepsilon\right)+P\left(\left|V_{n} h_{n}(b)\right| \geq \varepsilon\right) \\
& \leq \delta+P\left(\left|V_{n} h_{n}(b)\right| \geq \varepsilon,\left|h_{n}(b)\right| \leq \varepsilon / M\right)+\delta \\
& \leq P\left(\left|V_{n}\right| \geq M\right)+2 \delta \\
& \leq 3 \delta .
\end{aligned}
$$

Proof of Lemma 4.2. The differences $W_{n i}-W_{i}$ in eq. (4.4) and (4.1) can be expressed in terms of

$$
\begin{aligned}
\gamma_{i} & =\frac{\xi\left(Z_{i}\right) \delta_{i}}{\bar{G}_{n}\left(Z_{i}-\right)}-\frac{\xi\left(Z_{i}\right) \delta_{i}}{\bar{G}\left(Z_{i}\right)}, \\
\eta_{i} & =\frac{\bar{\delta}_{i}}{\bar{H}_{n}\left(Z_{i}-\right)} \psi_{n}\left(Z_{i}\right)-\frac{\bar{\delta}_{i}}{\bar{H}\left(Z_{i}\right)} \psi\left(Z_{i}\right), \\
\nu_{i} & =\int \psi_{n}(s) \frac{I\left[Z_{i} \geq s\right]}{\bar{H}_{n}^{2}(s-)} d H_{n}^{0}(s)-\int \psi(s) \frac{I\left[Z_{i} \geq s\right]}{\bar{H}^{2}(s)} d H^{0}(s),
\end{aligned}
$$

as

$$
W_{n i}-W_{i}=\gamma_{i}+\eta_{i}-\nu_{i} .
$$


Applying an elementary inequality $(a+b+c)^{2} \leq 3\left(a^{2}+b^{2}+c^{2}\right)$, we obtain

$$
\left(W_{n i}-W_{i}\right)^{2}=\left(\gamma_{i}+\eta_{i}-\nu_{i}\right)^{2} \leq 3\left(\gamma_{i}^{2}+\eta_{i}^{2}+\nu_{i}^{2}\right)
$$

The lemma will be proven by showing that the sample means of $\gamma_{i}^{2}, \eta_{i}^{2}$ and $\nu_{i}^{2}$ tend to zero in probability. The proofs will be presented in (A), (B) and (C) below.

(A) The sample mean of $\gamma_{i}^{2}$ is $o_{p}(1)$.

Proof: Let $G_{n}(x)$ be the K-M estimator defined in (2.3) and $b<b_{H}$. Then as $n \rightarrow \infty$,

$$
U_{n}=\sup _{s \leq b} \frac{\left|G_{n}(s-)-G(s)\right|}{\bar{G}_{n}(s)}=o_{p}(1), \quad V_{n}=\sup _{s \leq \max \left\{Z_{i}\right\}} \frac{\left|G_{n}(s-)-G(s)\right|}{\bar{G}_{n}(s-)}=O_{p}(1) .
$$

See Zhou (1992). To apply this result, we shall in the following proof split the integrals into two intervals $[0, b]$ and $\left(b, b_{H}\right]$ accordingly.

For any $b<b_{H}$, using $(2.2)$, we have

$$
\begin{aligned}
\frac{1}{n} \sum_{i=1}^{n} \gamma_{i}^{2} & =\int\left(\frac{\xi(s)}{\bar{G}_{n}(s-)}-\frac{\xi(s)}{\bar{G}(s)}\right)^{2} \mathrm{~d} H_{n}^{1}(s) \\
& \leq U_{n}^{2} \int_{0}^{b} \frac{\xi^{2}(s)}{\bar{G}^{2}(s)} \mathrm{d} H_{n}^{1}(s)+V_{n}^{2} \int_{b}^{b_{H}} \frac{\xi^{2}(s)}{\bar{G}^{2}(s)} \mathrm{d} H_{n}^{1}(s) \\
& =o_{p}(1) O_{p}(1)+O_{p}(1) h_{n}(b)=o_{p}(1), \text { as } n \rightarrow \infty,
\end{aligned}
$$

where $U_{n}^{2}$ and $V_{n}^{2}$ are given by (6.2) and

$$
h_{n}(b)=\int_{b}^{b_{H}} \frac{\xi^{2}(s)}{\bar{G}^{2}(s)} \mathrm{d} H_{n}^{1}(s) .
$$

Recall that $h_{n}(b)$ is explained in (3.12). It was shown that $h_{n}(b)$ satisfies the conditions in Lemma 3.2. The proof follows by invoking Lemma 3.2.

(B) The sample mean of $\eta_{i}^{2}$ is $o_{p}(1)$.

Proof: For $b<b_{H}$, define

$$
T_{n}(b, t]=\int_{b}^{t} \frac{\psi_{n}^{2}(s)}{\bar{H}_{n}^{2}(s-)} \mathrm{d} H_{n}^{0}(s), \quad S_{n}(b, t]=\int_{b}^{t} \frac{\psi^{2}(s)}{\bar{H}^{2}(s)} \mathrm{d} H_{n}^{0}(s) .
$$


Observe that

$$
\psi_{n}^{2}(s)=\left(\int_{u \geq s} \xi(u) \mathrm{d} F_{n}(u)\right)^{2} \leq \bar{F}_{n}(s-) \int_{u \geq s} \xi^{2}(u) \mathrm{d} F_{n}(u),
$$

and $F_{n}$ and $G_{n}$ have no common jumps. It follows that

$$
\begin{aligned}
T_{n}\left(b, b_{H}\right] & \leq \int_{b}^{b_{H}}\left(\int_{u \geq s} \xi^{2}(u) \mathrm{d} F_{n}(u)\right) \bar{F}_{n}^{2}(s-) \frac{\mathrm{d} G_{n}(s)}{\bar{H}_{n}^{2}(s-)} \\
& \leq \int_{b}^{b_{H}}\left(\int_{u \geq s} \xi^{2}(u) \mathrm{d} F_{n}(u)\right) \mathrm{d}\left(\frac{1}{\bar{G}_{n}(s)}\right) \\
& \leq \lim _{s \rightarrow b_{H}} \frac{1}{\bar{G}_{n}(s)} \int_{s}^{b_{H}} \xi^{2}(u) \mathrm{d} F_{n}(u)+\int_{b}^{b_{H}} \frac{\xi^{2}(s)}{\bar{G}_{n}(s-)} \mathrm{d} F_{n}(s) \\
& \leq 2 \int_{b}^{b_{H}} \frac{\xi^{2}(s)}{\bar{G}_{n}(s-)} \mathrm{d} F_{n}(s) \\
& \leq 2 \int_{b}^{b_{H}}\left(\frac{1}{\bar{G}_{n}(s-)}-\frac{1}{\bar{G}(s)}+\frac{1}{\bar{G}(s)}\right)^{2} \xi^{2}(s) \mathrm{d} H_{n}^{1}(s) \\
& =o_{p}(1)+O_{p}(1) h_{n}(b) .
\end{aligned}
$$

The first inequality follows from (2.6) the second and the third from integration by parts, the fifth from (2.5) and the last equality from $(a+b)^{2} \leq 2\left(a^{2}+b^{2}\right),(6.3)$ and $(6.4)$.

By the same token, we conclude that $S_{n}\left(b, b_{H}\right]=o_{p}(1)+O_{p}(1) h_{n}(b)$.

Write

$$
\xi=\xi^{+}-\xi^{-}, \psi_{n}(x)=\int_{s \geq x} \xi^{+}(s) \mathrm{d} F_{n}(s)-\int_{s \geq x} \xi^{-}(s) \mathrm{d} F_{n}(s),
$$

where $\xi^{+}$and $\xi^{-}$are the positive and negative part of $\xi$. Define monotone functions:

$$
\psi_{n}^{ \pm}(x)=\int_{s \geq x} \xi^{ \pm}(s) \mathrm{d} F_{n}(s), \psi^{ \pm}(x)=\int_{s \geq x} \xi^{ \pm}(s) \mathrm{d} F(s) .
$$

$\psi_{n}^{ \pm}(x)$ converges to $\psi^{ \pm}(x)$ almost surely for $x \in\left[0, b_{H}\right]$ as shown by Stute \& Wang (1993). Furthermore, by Lemma 4.1 , the convergence is uniform on $\left[0, b_{H}\right]$.

From these we conclude the uniform convergence of $\psi_{n}$ to $\psi$,

$$
\sup _{0 \leq x \leq b_{H}}\left|\psi_{n}(x)-\psi(x)\right|=o(1), \text { a.s.. }
$$


Therefore, for $b<b_{H}$,

$$
\sup _{s \leq b}\left(\frac{\psi_{n}(s)}{\bar{H}_{n}(s-)}-\frac{\psi(s)}{\bar{H}(s)}\right)^{2} \rightarrow 0, \text { a.s.. }
$$

Applying $(a+b)^{2} \leq 2 a^{2}+2 b^{2}$ and Lemma 3.2, we have

$$
\begin{aligned}
\frac{1}{n} \sum_{i=1}^{n} \eta_{i}^{2} & =\int_{0}^{b}+\int_{b}^{b_{H}}\left(\frac{\psi_{n}(s)}{\bar{H}_{n}(s-)}-\frac{\psi(s)}{\bar{H}(s)}\right)^{2} \mathrm{~d} H_{n}^{0}(s) \\
& \leq \int_{0}^{b}\left(\frac{\psi_{n}(s)}{\bar{H}_{n}(s-)}-\frac{\psi(s)}{\bar{H}(s)}\right)^{2} \mathrm{~d} H_{n}^{0}(s)+2 T_{n}\left(b, b_{H}\right]+2 S_{n}\left(b, b_{H}\right] \\
& =o_{p}(1)+O_{p}(1) h_{n}(b)+O_{p}(1) h_{n}(b)=o_{p}(1) .
\end{aligned}
$$

(C) The sample mean of $\nu_{i}^{2}$ is $o_{p}(1)$.

Proof: Write, for $0 \leq a<t$,

$$
B_{n}(a, t]=\int_{a}^{t} \frac{\psi_{n}(s) \mathrm{d} H_{n}^{0}(s)}{\bar{H}_{n}^{2}(s-)}, \quad D(a, t]=\int_{a}^{t} \frac{\psi(s) \mathrm{d} H^{0}(s)}{\bar{H}^{2}(s)} .
$$

Then, for $b<b_{H}$, we have

$$
\begin{aligned}
\Delta_{n}^{2} & \equiv \int_{b}^{b_{H}} B_{n}^{2}(b, t] \mathrm{d} H_{n}(t)=\int_{b}^{b_{H}} B_{n}^{2}(b, t] \mathrm{d}\left(-\bar{H}_{n}(t)\right) \\
& \leq \bar{H}_{n}(b) B_{n}^{2}(b, b]+2 \int_{b}^{b_{H}} \bar{H}_{n}(t-) B_{n}(b, t] \frac{\psi_{n}(t)}{\bar{H}_{n}^{2}(t-)} \mathrm{d} H_{n}^{0}(t) \\
& \leq 0+2\left(\int_{b}^{b_{H}} B_{n}^{2}(b, t] \mathrm{d} H_{n}(t)\right)^{1 / 2}\left(\int_{b}^{b_{H}} \frac{\psi_{n}^{2}(t)}{\bar{H}_{n}^{2}(t-)} \mathrm{d} H_{n}^{0}(t)\right)^{1 / 2} \\
& =\Delta_{n} 2\left[T_{n}\left(b, b_{H}\right)\right]^{1 / 2} .
\end{aligned}
$$

The second term in the first inequality is obtained using the Lebesgue-Stieltjes integration by parts.

Applying (6.5), we get

$$
\int_{b}^{b_{H}} B_{n}^{2}(b, t] \mathrm{d} H_{n}(t)=\Delta_{n}^{2} \leq 4 T_{n}\left(b, b_{H}\right]=o_{p}(1)+O_{p}(1) h_{n}(b) .
$$

Similarly, for $S\left(b, b_{H}\right]=\mathrm{E} S_{n}\left(b, b_{H}\right]$, we have

$$
\int_{b}^{b_{H}} D^{2}(b, t] \mathrm{d} H_{n}(t) \leq 4 S\left(b, b_{H}\right]+o_{p}(1)=o_{p}(1)+O_{p}(1) h_{n}(b) .
$$


Applying the uniform convergence of $\psi_{n}$ to $\psi$ (see(6.6)), we conclude that for any $b<b_{H}$, with probability $1, B_{n}(0, t] \rightarrow D(0, t]$ uniformly on $[0, b]$. It follows that $B_{n}(0, b] \rightarrow D(0, b]$, and

$$
\begin{aligned}
\frac{1}{n} \sum_{i=1}^{n} \nu_{i}^{2} & =\int_{0}^{b}+\int_{b}^{b_{H}}\left(B_{n}(0, t]-D(0, t]\right)^{2} \mathrm{~d} H_{n}(t) \\
& =o_{p}(1)+\int_{b}^{b_{H}}\left(B_{n}(0, b]-D(0, b]+B_{n}(b, t]-D(b, t]\right)^{2} \mathrm{~d} H_{n}(t) \\
& \leq o_{p}(1)+4 \int_{b}^{b_{H}} B_{n}^{2}(b, t] \mathrm{d} H_{n}(t)+4 \int_{b}^{b_{H}} D^{2}(b, t) \mathrm{d} H_{n}(t) \\
& =o_{p}(1)+O_{p}(1) h_{n}(b)+O_{p}(1) h_{n}(b)=o_{p}(1) .
\end{aligned}
$$

Now, we prove result (2) of the lemma. Introduce $A_{n i}=W_{i} \bar{H}_{n}\left(Z_{i}-\right) \bar{H}\left(Z_{i}\right)$. From (6.1) we get

$$
\left(V_{n i}-A_{n i}\right)^{2} \leq 3\left(\gamma_{i}^{2}+\eta_{i}^{2}+\nu_{i}^{2}\right) \bar{H}_{n}^{2}\left(Z_{i}-\right) \bar{H}^{2}\left(Z_{i}\right) .
$$

Similar to (6.3) and (6.7), we have

$$
\begin{aligned}
& \frac{1}{n} \sum_{i=1}^{n} \gamma_{i}^{2} \bar{H}_{n}^{2}\left(Z_{i}-\right) \bar{H}^{2}\left(Z_{i}\right) \\
= & \int_{0}^{b_{H}}\left(\frac{\xi(s)}{\bar{G}_{n}(s-)}-\frac{\xi(s)}{\bar{G}(s)}\right)^{2} \bar{H}_{n}^{2}(s-) \bar{H}^{2}(s) \mathrm{d} H_{n}^{1}(s)=o(1), \text { a.s. } \\
& \frac{1}{n} \sum_{i=1}^{n} \eta_{i}^{2} \bar{H}_{n}^{2}\left(Z_{i}-\right) \bar{H}^{2}\left(Z_{i}\right) \\
= & \int_{0}^{b_{H}}\left(\frac{\psi_{n}(s)}{\bar{H}_{n}(s-)}-\frac{\psi(s)}{\bar{H}(s)}\right)^{2} \bar{H}_{n}^{2}(s-) \bar{H}^{2}(s) \mathrm{d} H_{n}^{0}(s)=o(1), \text { a.s.. }
\end{aligned}
$$

Since for any $b<b_{H}$, with probability 1 ,

$$
\left|B_{n}(0, t]-D(0, t]\right| \bar{H}_{n}(t-) \bar{H}(t) \rightarrow 0
$$

uniformly on $[0, b]$, and

$$
\sup _{t \leq b_{H}}\left|B_{n}(0, t]-D(0, t]\right| \bar{H}_{n}(t-) \bar{H}(t)
$$


is bounded by some constant, it follows that

$$
\begin{aligned}
& \frac{1}{n} \sum_{i=1}^{n} \nu_{i}^{2} \bar{H}_{n}^{2}\left(Z_{i}-\right) \bar{H}^{2}\left(Z_{i}\right) \\
= & \int_{0}^{b_{H}}\left[\left(B_{n}(0, t]-D(0, t]\right) \bar{H}_{n}(t-) \bar{H}(t)\right]^{2} \mathrm{~d} H_{n}(t)=o(1), \text { a.s.. }
\end{aligned}
$$

Now we get

$$
\frac{1}{n} \sum_{i=1}^{n}\left(V_{n i}-A_{n i}\right)^{2} \leq 3 \frac{1}{n} \sum_{i=1}^{n}\left(\gamma_{i}^{2}+\eta_{i}^{2}+\nu_{i}^{2}\right) \bar{H}_{n}^{2}\left(Z_{i}-\right) \bar{H}^{2}\left(Z_{i}\right)=o(1), \text { a.s. }
$$

At last, we have

$$
\begin{aligned}
\frac{1}{n} \sum_{i=1}^{n}\left(V_{n i}-V_{i}\right)^{2} & \leq \frac{2}{n} \sum_{i=1}^{n}\left(V_{n i}-A_{n i}\right)^{2}+\frac{2}{n} \sum_{i=1}^{n}\left(A_{n i}-V_{i}\right)^{2} \\
& =o(1)+\frac{2}{n} \sum_{i=1}^{n}\left(H\left(Z_{i}\right)-H_{n}\left(Z_{i}-\right)\right)^{2} W_{i}^{2} \bar{H}^{2}\left(Z_{i}\right) \\
& =o(1), \text { a.s. }
\end{aligned}
$$

Proof of Lemma 4.3. Since $W_{i}$ are i.i.d. random variables with zero mean and finite variance $\sigma^{2}$, hence $\max _{1 \leq i \leq n}\left|W_{i}\right|=o_{p}(\sqrt{n})$. It follows from Lemma 4.2 that

$$
\begin{aligned}
\max _{1 \leq i \leq n}\left|W_{n i}\right| & \leq\left(\max _{1 \leq i \leq n}\left|W_{n i}-W_{i}\right|^{2}\right)^{1 / 2}+\max _{1 \leq i \leq n}\left|W_{i}\right| \\
& \leq \sqrt{n}\left(\frac{1}{n} \sum_{i=1}^{n}\left(W_{n i}-W_{i}\right)^{2}\right)^{1 / 2}+o_{p}(\sqrt{n}) \\
& =o_{p}(\sqrt{n}) .
\end{aligned}
$$

Note that $W_{n i}^{2}$ is bounded by

$W_{i}^{2}+\left(W_{i}-W_{n i}\right)^{2}-2\left|W_{i}\left(W_{i}-W_{n i}\right)\right| \leq W_{n i}^{2} \leq W_{i}^{2}+\left(W_{n i}-W_{i}\right)^{2}+2\left|W_{i}\left(W_{i}-W_{n i}\right)\right|$

By Lemma 4.2, we get

$$
\lim _{n \rightarrow \infty} \frac{1}{n} \sum_{i=1}^{n} W_{n i}^{2}=\sigma^{2}+o_{p}(1)
$$

The last result follows from Theorem 3.1, $\xi(x)=g\left(x, \theta_{0}\right)$ and (4.6). 


\section{References}

[1] Chen, K. and Lo, S-H. (1997). On the rate of uniform convergence of the product-limit estimator: strong and weak laws. Ann. Statist. 25. 1050-1087.

[2] Chen, S. X. (1994). Empirical likelihood confidence intervals for linear regression coefficients. J. Multivariate Anal. 49. 24-40.

[3] DiCiccio, T. J., Hall, P. and Romano, J.P. (1991). Empirical likelihood is Bartlett-correctable. Ann. Statist. 19. 1053-1061.

[4] He, S. Y. and Huang, X. (2003). Central limit theorem of linear regression model under right censorship. Science in China. 46. 600-610.

[5] Hjort, N. L., McKeague, I. W., and van Keilegom, I. (2009): Extending the scope of empirical likelihood. Ann. Statist. 37, 1079-1111

[6] Li, G. and Wang, QI-HuA (2003) Empirical likelihood methods for linear regression analysis of right censored data, Statist. Sinica 13 51-68.

[7] Lo, S-H. and Singh, P. (1986). The product-limit estimator and the bootstrap: some asymptotic representations. Probab. Theory Related Fields. 71. 455-465.

[8] Owen, A. (1988). Empirical likelihood ratio confidence intervals for single functional. Biometrika. 75. 237-249.

[9] Owen, A. (2001). Empirical likelihood. Chapman and Hall, London.

[10] QIN, G. S. and Zhao, Y. C. (2007). Empirical likelihood inference for the mean residual life under random censorship. Statist. Probab. Lett. 77. 549-557.

[11] REN, J. J. (2008). Weighted empirical likelihood in some two-sample semiparametric models with various types of censored data. Ann. Statist. 36. 147-166. 
[12] Shorack, G. and Wellner, J. (1986). Empirical Processes with Applications to Statistics. Wiley, New York.

[13] Stute, W. and Wang, J. L. (1993). The strong law under random censorship. Ann. Statist. 21. 1591-1607.

[14] Stute, W. (1996). The Jackknife estimate of variance of a Kaplan-Meier integral. Ann. Statist. 24. 2679-2704.

[15] Tsiatis A. A. (2006). Semiparametric Theory and Missing Data. New York: Springer.

[16] Van Der VaArt, A. W. (1998). Asymptotic Statistics. Cambridge University Press.

[17] VARDi Y. (1982). Nonparametric estimation in the presence of length bias. Ann. Statist. 10. 616-620.

[18] Wang, Q. H. and Jing, B. Y. (2001). Empirical likelihood for a class of functions of survival distribution with censored data. Ann. Inst. Statist. Math. 53. 517-527.

[19] YAng, G. (1997). The Kaplan-Meier estimator. Encyclopedia of Statistical Sciences, update Vol. 1, Wiley, 334-343.

[20] YANG, S. (1994). A central limit theorem for functionals of the Kaplan-Meier estimator. Statist. Probab. Lett. 21. 337-345.

[21] Zhou, M. (1992). Asymptotic normality of the 'synthetic data' regression estimator for censored survival data. Ann. Statist. 20. 1002-1021. 